\title{
Structure factor and thermodynamics of rigid dendrimers in solution
}

\author{
L. Harnau ${ }^{a}$, S. Rosenfeldt ${ }^{b}$, and M. Ballauff ${ }^{b}$ \\ ${ }^{a}$ Max-Planck-Institut für Metallforschung, Heisenbergstr. 3, D-70569 Stuttgart, Germany, \\ and Institut für Theoretische und Angewandte Physik, \\ Universität Stuttgart, Pfaffenwaldring 57, D-70569 Stuttgart, Germany \\ ${ }^{b}$ Physikalische Chemie I, University of Bayreuth, D-95440 Bayreuth, Germany
}

(Dated: November 20, 2018)

\begin{abstract}
The "polymer reference interaction site model" (PRISM) integral equation theory is used to determine the structure factor of rigid dendrimers in solution. The theory is quite successful in reproducing experimental structure factors for various dendrimer concentrations. In addition, the structure factor at vanishing scattering vector is calculated via the compressibility equation using scaled particle theory and fundamental measure theory. The results as predicted by both theories are systematically smaller than the experimental and PRISM data for platelike dendrimers.
\end{abstract}

PACS numbers: $61.20 . \mathrm{Gy}, 82.70 . \mathrm{Dd}, 83.70 . \mathrm{Hq}$

\section{INTRODUCTION}

Dendrimers are synthetic macromolecules with defined architecture that are synthesized by iterative controlled reaction steps. Starting from a focal unit, subsequent generations are connected to this initial core which results in a tree-like structure $1,2,3,4$. Today it is wellunderstood that dendrimers composed of flexible units adopt a so-called dense core structure, that is, the average segmental density has its maximum at the center of the molecule $2,3,4$. This is easily derived from the fact that flexible dendrimers can assume a great number of conformations in which the terminal groups can fold back. Hence these flexible dendrimers do not exhibit a welldefined surface given by the terminal groups of the last generation. The average density profile thus derived can be used to calculate the interaction of flexible dendrimers in solution. Thus these structure are well-understood by now ${ }^{\underline{5}}$.

Much less attention has been paid to dendrimers consisting of rigid units $6.7,8,9,10,11,12,13,14$. Figure1(a) shows the chemical structure of such a rigid dendrimer which is solely composed of stiff units $6,8,9,10$. While the dendritic scaffold of flexible dendrimers in solution can adopt a large number of conformations which follow from rotations about various bonds, dendrimers consisting of such rigid units exhibit a rather well defined structure in solution. This fact has been shown recently by small-angle neutron scattering (SANS)표.,14. Hence, these systems may serve as model systems for interacting monodisperse particles in statistical physics.

Here we present a comprehensive discussion of the interaction of rigid dendrimers in solution. Two systems involving rigid dendrimers are examined in the present paper, namely polyphenylene dendrimers of the fourth generation in solution (see Fig. 1(a)) and stilbenoid dendrimers of the third generation in solution (see Fig.1(b)). The problem to be addressed is that of structural properties over a range of dendrimer concentrations. In general, the effect of mutual interaction of dissolved species with number density $\rho=N / V$ in a scattering experiment can be embodied in the structure factor $S(q, \rho)$ defined as

$$
I(q, \rho)=\frac{N}{V} I_{0}(q) S(q, \rho)=\frac{N}{V} V_{p}^{2}(\Delta \bar{\rho})^{2} P(q) S(q, \rho)
$$

where $I_{0}(q)$ is the scattering intensity of the single particle, $V_{p}$ the volume of the particle, $P(q)$ the form factor (normalized to unity for $q=0$ ) and $q$ is the magnitude of the scattering vector $(q=(4 \pi / \lambda) \sin (\theta / 2), \lambda$ : wavelength of radiation, $\theta$ : scattering angle). $\Delta \bar{\rho}$ is the contrast of the solute resulting from the difference of the average scattering length density and the scattering length density of the solvent. Up to now, mutual interaction has only been addressed because scattering experiments require finite concentrations in order to obtain data with a reasonable statistics $7,8,14$. Hence, the effect of mutual interaction of the dissolved dendrimers has only been considered when extrapolating the data to vanishing concentrations. Details of such a data evaluation may be found

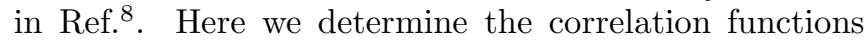
and structure factors $S(q, \rho)$ within the framework of an interaction site model and compare these results to experimental data $7,8,14$. Moreover, the validity of the predictions of both scaled particle theory and fundamental measure theory are investigated. A systematic comparison is made between the results of the theories and experimental data for two differently shaped dendrimers in solution, in order to quantify the influence of the shape of the particles on the quality of the predictions of the theories. We demonstrate that rigid dendrimers present a new class of strictly monodisperse model colloids whose interaction can be conveniently studied in solution. In this way we show that these systems allow us to re-consider the basic statistical mechanics of rigid objects in solution and to compare theory and experiment for the first time in a rigorous fashion.

The paper is organized as follows: In the next section we briefly recapitulate the analytical models necessary to calculate the scattering intensity for a system of interacting rigid bodies. Thereafter the theory will be compared to experimental results obtained for the rigid dendrimers shown in Figs. 1 (a) and (b). In section IV these results 
(a)

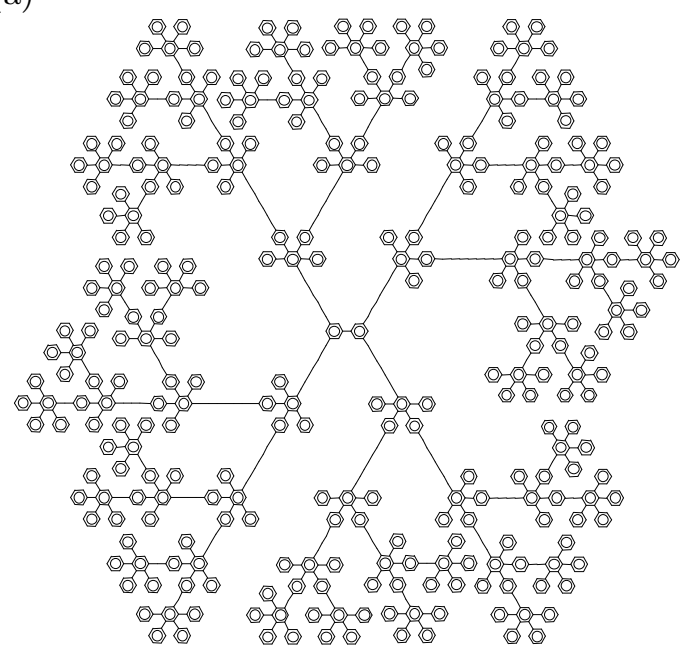

(b)

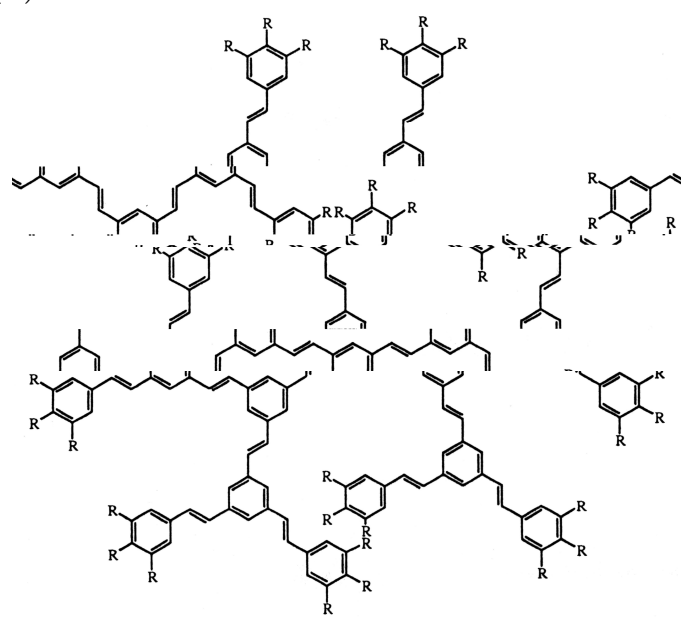

FIG. 1: (a) Chemical structure of the polyphenylene dendrimer of the fourth generation. (b) Chemical structure of the stilbenoid dendrimer of the third generation $\left(\mathrm{R}=\mathrm{OC}_{6} \mathrm{H}_{13}\right)$.

are compared to the fundamentals of the thermodynamics of rigid bodies. Special attention is paid to a discussion of exact virial coefficients. A brief conclusion will summarize the results at the end.

\section{RISM AND PRISM THEORIES}

The systems under investigation are solutions. However, in view of the mesoscopic scale of the particles, the solvent will be considered as a structureless continuum. Spatial pair correlations of an isotropic fluid of identical particles, each carrying $n$ distinct interaction sites, are characterized by a set of intermolecular site-site total correlation functions $h_{i j}(r, \rho)$, where the indices $i$ and $j$ run over sites on each of two particles and $\rho$ is the particle number density. These functions are related to a set of intermolecular site-site direct correlation functions $c_{i j}(r, \rho)$ by the generalized Ornstein-Zernike relations of the "reference interaction site model" (RISM), which in Fourier space read 16,17

$h_{i j}(q, \rho)=\sum_{m, o=1}^{n} \omega_{i m}(q, \rho) c_{m o}(q, \rho)\left(\omega_{o j}(q, \rho)+\rho h_{o j}(q, \rho)\right)$,

where the $\omega_{i j}(q, \rho)$ are the Fourier transforms of the intramolecular correlation functions. The set of generalized Ornstein-Zernike equations must be supplemented by a set of closure relations. If the interaction sites are simply the centers of exclusion spheres of diameter $d$, to account for steric effects, a convenient closure is the Percus-Yevick approximation 16.18

$$
h_{i j}(r, \rho)=-1, r \leq d, \quad c_{i j}(r, \rho)=0, r>d .
$$

The experimentally accessible structure factor $S(q, \rho)$ is defined as

$$
S(q, \rho)=1+\rho \frac{h(q, \rho)}{P(q, \rho)},
$$

where

$$
h(q, \rho)=\frac{1}{n^{2}} \sum_{m, o=1}^{n} h_{m o}(q, \rho)
$$

is the particle-averaged total correlation function. The particle-averaged intramolecular correlation function

$$
P(q, \rho)=\frac{1}{n^{2}} \sum_{m, o=1}^{n} \omega_{m o}(q, \rho)
$$

characterizes the geometry of the distribution of the sites, and hence the geometric shape of the particles. While the particle-averaged intramolecular correlation function accounts for the interference of radiation scattered from different parts of the same particle in a scattering experiment, the local order in the fluid is characterized by $h(q, \rho)$ or $S(q, \rho)$.

The RISM has been proved to be a successful theory of the pair structure of many molecular fluids (for a review see Ref. $\underline{19}$ ). In the case of macromolecular and colloidal systems, with very large numbers of interaction sites, the number of coupled RISM equations becomes intractable, and a considerable simplification follows from the assumption that the direct correlation functions $c_{i j}(q, \rho)$ are independent of the indices $i$ and $j$. This leads to the "polymer reference interaction site model" (PRISM) theory first applied by Schweizer and Curro to long flexible polymers ${ }^{20}$. PRISM neglects end effects in that case. The resulting single generalized Ornstein-Zernike equation of the PRISM reads

$$
h(q, \rho)=c(q, \rho) P^{2}(q, \rho)+\rho c(q, \rho) h(q, \rho) P(q, \rho),
$$

where $c(q, \rho)=\sum_{m, o=1}^{n} c_{m o}(q, \rho)$. The PRISM integral equation theory has been successfully applied to various systems, such as rodlike viruses ${ }^{21,22,23,24}$, platelike 


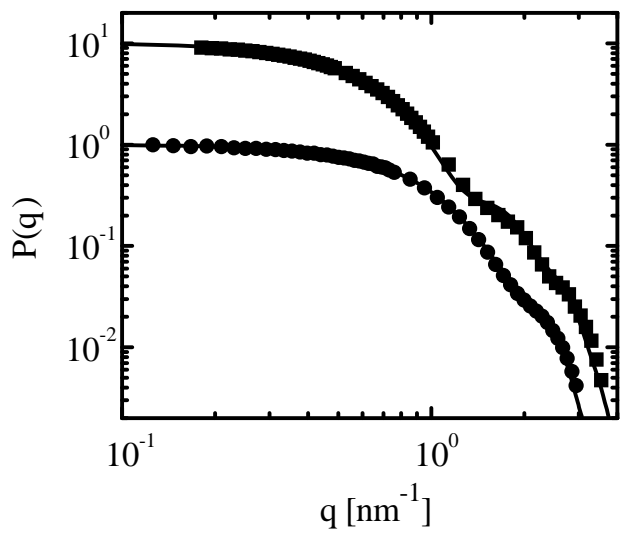

FIG. 2: The form factor $P(q)=P(q, \rho \rightarrow 0)$ of polyphenylene dendrimers of the fourth generation ${ }^{8}$ (squares and upper line) and stilbenoid dendrimers of the third generation ${ }^{14}$ (circles and lower line) as obtained by small angle neutron scattering (symbols). The lines represent the calculated form factors. For reasons of clarity, the upper data set and line have been shifted up.

colloids $25,26,27$ and dendrimers ${ }^{14}$, polymers 28,29 , mixtures of spherical colloids and semiflexible polymers ${ }^{30}$, and bottlebrush polymers 31 . Moreover, it has been demonstrated recently that the simpler PRISM theory yields results in good agreement with the more elaborate RISM calculations for lamellar colloids $\underline{32}$.

For flexible polymers or dendrimers the particleaveraged intramolecular correlation function $P(q, \rho)$ depends on the particle number density and follows from a statistcal average over particle configurations. In the limit $\rho \rightarrow 0$ the particle-averaged intramolecular correlation function reduces to the form factor $P(q) \equiv P(q, \rho \rightarrow 0)$. In the case of rigid particles $P(q, \rho)$ is independent of the particle number density because the particles are not deformed due to intermolecular interactions for typical concentrations in the fluid state.

\section{RIGID POLYPHENYLENE AND STILBENOID DENDRIMERS IN SOLUTION}

\section{A. Form factors}

It has been demonstrated that the building units of the dendritic scaffold of polyphenylene dendrimers of the fourth generation (see Fig. 11(a)) are rather well-localized and no back folding of the terminal groups occurs 7.8 . A general feature of the polyphenylene building block is its finite angle formed by two subsequent phenyl groups ${ }^{9.10}$. This is due to the strong repulsion between their ohydrogen atoms that prevent a co-planar conformation. As a consequence, the polyphenylene dendrimers exhibit a three-dimensional structure which has been found by SANS7,, .

Such steric hindrance does not exists in dendritic scaf- folds set up from stilbenoid units11,12,13. Starting from a central phenyl group all subsequent generations are built up by trans-stilben units, only the terminal groups are substituted by hexyloxy groups in order to ensure better solubility in common solvents such as toluene (see Fig. 1 (b)). Full conjugation in trans-stilbene can be achieved in the completely planar conformation. However, the potential energy for a slight torsion around the single bonds is low in the ground state of trans-stilbene. Both molecular modeling $\underline{12}$ and a small-angle scattering study $\underline{14}$ have demonstrated that a stilbenoid dendrimer of the third generation exhibits a relatively compact platelike structure.

The measured and calculated form factors $P(q)$ of both polyphenylene dendrimers of the fourth generation and stilbenoid dendrimers of the third generation are shown in Fig. 2, The form factor of the stilbenoid dendrimers agrees with the one calculated numerically for a circular platelet with a radius $R=2.4 \mathrm{~nm}$ and thickness $L=1.8 \mathrm{~nm}$ according to

$$
P(q)=4 \int_{0}^{1} d x \frac{J_{1}^{2}\left(q R \sqrt{1-x^{2}}\right)}{\left(q R \sqrt{1-x^{2}}\right)^{2}} \frac{\sin ^{2}(q L x / 2)}{(q L x / 2)^{2}},
$$

where $J_{1}(x)$ denotes the cylindrical Bessel function of first-order ${ }^{15}$. Equation (7) follows from Eq. (5) by replacing the double sums by double integrals corresponding to a continuous distribution of interaction sites $(n \rightarrow$ $\infty)$ and by using $\omega_{m o}(q, \rho)=\sin \left(q l_{m o}\right) /\left(q l_{m o}\right)$, where $l_{m o} \rightarrow 0$ is the bond length between sites $m$ and $o$ on the same cylindrical particle.

The polyphenylene dendrimers were modelled by a central unit consisting of two small spheres (diameter: $0.4 \mathrm{~nm}$ ). This describes the central biphenyl unit (see Fig. 3). The four dendrons were mimicked by eight spheres (diameter: $0.8 \mathrm{~nm}$ ) with a center to center distance of $0.87 \mathrm{~nm}$. This distance is the approximate length of the two phenyl groups that connect two subsequent shells. Each sphere comprises five benzene rings that constitute a branching group. The endgroups are made of an equal number of phenyl rings. The form factor of the polyphenylene dendrimers has been modelled by taking an average over approximately 500 conformers generated by randomly choosing the torsion angles for each dendron (see Ref $\stackrel{8}{8}$ for further details). The good agreement between the experimental and theoretical results (Fig. 2) demonstrates that a full understanding of the spatial structure of both polyphenylene dendrimers of the fourth generation and stilbenoid dendrimers of the third generation has been achieved ${ }^{8,14}$. The ability to construct coarse-grained models in such a way as to reproduce experimentally determined form factors is important as it allows one to quickly study within PRISM theory various material systems based on input of a small amount of intramolecular information. 


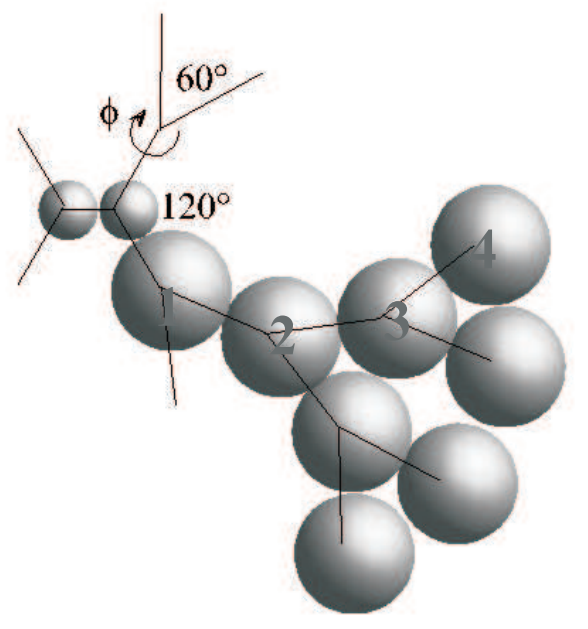

FIG. 3: Modeling of the the form factor $P(q)$ of the polyphenylene dendrimer of the fourth generation. The dendritic scaffold is set up of a central unit consisting of two small spheres (distance: $0.4 \mathrm{~nm}$ ) describing the central biphenyl unit. The four dendrons were modeled by 8 spheres (distance: $0.87 \mathrm{~nm}$ ), respectively, having a diameter of $0.8 \mathrm{~nm}$. Both parameters were used as a fitting parameter. For the sake of clarity the size of the spheres has been depicted slightly smaller. The angles between the different units is indicated in the graph. The torsional angle was chosen at random for each dendron.

\section{B. Structure factors}

In Figs. 4 (a) and (b) the experimental structure factors $S(q, \phi)$ are compared to the results of the integral equation theory for the PRISM. We have used the calculated form factors $P(q)$ (see the solid lines in Fig. 2) as input into the generalized Ornstein-Zernike equation, i.e., $P(q, \phi)=P(q)$ in Eqs. (3) and (6). The particle number density is given by $\rho=\phi / V_{p}$, where $\phi$ is the volume fraction and $V_{p}$ is the volume of an individual particle. The generalized Ornstein-Zernike equation is solved numerically together with the Percus-Yevick closure. From Fig. 4 it is apparent that the PRISM integral equation theory is rather accurate. The magnitude and the scattering vector range of the suppression of $S(q, \rho)$, i.e., the deviations from the value 1 at small scattering vectors, are characteristic for the size and the shape of the dendrimers as well as the volume fraction. The experimentally observed small upturns of $S(q, \rho)$ at low scattering vectors $q$ for polyphenylene dendrimers in solution (Fig. 4 (a)) indicate the presence of a small amount of aggregates due to attractive interactions which are not taken into account in the theoretical calculations. On the basis of our experience with both PRISM and RISM we expect that the results of the integral equation theory for the RISM would lead to very similar results, provided the same form factors are used ${ }^{32}$.
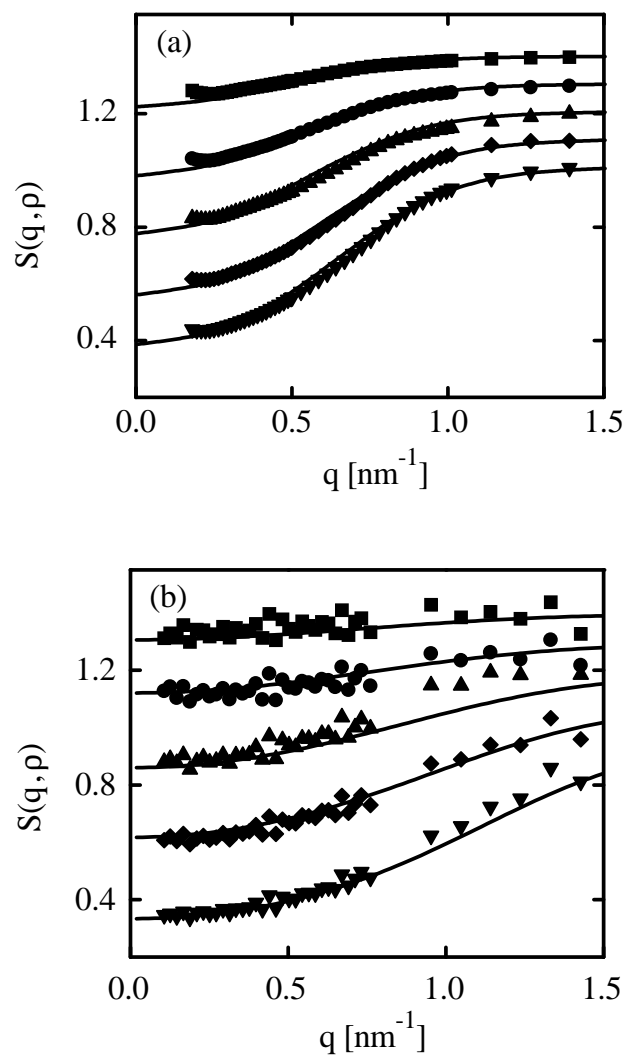

FIG. 4: Experimentally determined structure factors for polyphenylene dendrimers of the fourth generation 8 in (a) and stilbenoid dendrimers of the third generation ${ }^{14}$ in (b) together with the results of the theoretical predictions of the PRISM integral equation theory (Eqs. (3) and (6)). The volume fraction of the dendrimers increases from top to bottom: $\phi=0.016,0.032,0.046,0.065,0.08$ in (a); $\phi=$ $0.009,0.019,0.039,0.061,0.1$ in (b). For reasons of clarity, the upper data sets and lines have been shifted up by $0.1,0.2$, $0.3,0.4$, respectively.

\section{THERMODYNAMIC PROPERTIES}

The structure factor provides a direct link with thermodynamics via the compressibility equation ${ }^{18}$

$$
\lim _{q \rightarrow 0} S(q, \rho)=\rho k_{B} T \kappa_{T}(\rho),
$$

where $\kappa_{T}(\rho)$ is the isothermal compressibility. The osmotic pressure $P(\rho)$ (equation of state) then follows from

$$
\frac{P(\rho)}{k_{B} T}=\int_{0}^{\rho} d \rho^{\prime} S^{-1}\left(q=0, \rho^{\prime}\right) .
$$

Various attempts have been made to develop accurate theories for the equation of state of fluids consisting of non-spherical particles:

(a) Scaled particle theory ${ }^{33,34,35}$, which is very successful for hard sphere fluids, has been extended to prolate and oblate ellipsoids of revolution ${ }^{36}$, however 
with moderate success when gauged against Monte Carlo simulations ${ }^{37}$. Recently, it has been shown 38 that the results of scaled particle theory ${ }^{38,39.40}$ for platelike particles or the closely related model of hard cut spheres are in disagreement with computer simulation data $41,42,43,44,45,46,47$.

(b) Onsager theory ${ }^{48.49}$, based on the second viral coefficient alone, can be "rescaled" 50,51,52. Also this semiempirical procedure leads to reasonably good results for rodlike particles, it is much less satisfactory for platelike particles 53 .

(c) Many theoretical studies on hard sphere fluids and depletion agents use the so-called free volume theory ${ }^{54}$, in which the free volume accessible to a single particle plays a major role. Recently, this free volume theory has been studied within a fundamental measure theory 38 . However, it has been demonstrated that the resulting third virial coefficients of the equation of state for both hard cylinders and hard cut spheres differ from computer simulation results (see tables I and II in Ref ${ }^{38}$ ). Theoretical approaches based on fundamental measure theory do not yield correct third virial coefficients and equation of states due to the occurrence of so-called "lost cases", i.e., the fact configurations of three particles with pairwise overlap but no triple overlap do not contribute to thermodynamic properties (see, e.g., Refs $\stackrel{55,56}{5}$ ).

These earlier theoretical and computer simulation studies demonstrate that the understanding of thermodynamic properties of non-spherical particles needs to be improved. Here we model the measured inverse structure factor $S^{-1}(q=0, \rho)$ extrapolated to vanishing scattering vectors of stilbenoid dendrimers of the third generation in terms of the so-called $y 3$-expansion 57,58

$$
\begin{aligned}
S^{-1}(q=0, \rho) & =\frac{1+2\left(B_{2}-2\right) \phi+\left(3 B_{3}-8 B_{2}+6\right) \phi^{2}}{(1-\phi)^{4}} \\
& \approx 1+2 B_{2} \phi+3 B_{3} \phi^{2}+O\left(\phi^{3}\right) .
\end{aligned}
$$

The $y 3$ theory reproduces the exact second and third virial coefficients, $B_{2}$ and $B_{3}$, respectively. However, its practical applicability is limited due to the difficult numerical evaluation of the third virial coefficient $B_{3}$ in the case of non-spherical particles 59 , while the second virial coefficient $B_{2}$ for an isotropic hard convex body fluid is known exactly (see Ref $\underline{60}^{6}$ and references therein):

$$
B_{2}=\frac{1}{V_{p}}\left(V_{p}+A_{p} \tilde{R_{p}}\right) .
$$

Here $A_{p}$ and $\tilde{R}_{p}=(1 / 4 \pi) \int d A_{p} H_{p}$ are the surface area and the mean radius, respectively, where the local mean curvature is denoted as $H_{p}$. For a circular platelet of radius $R$ and thickness $L$ one has $V_{p}=\pi R^{2} L$, $A_{p}=2 \pi R(R+L)$, and $\tilde{R}_{p}=\pi R / 4+L / 4$. For platelike stilbenoid dendrimers of the third generation the second virial coefficient $B_{2}=5.54$ as calculated from Eq. (12) with $R=2.4 \mathrm{~nm}$ and $L=1.8 \mathrm{~nm}$ agrees with the experimentally determined second virial coefficient.

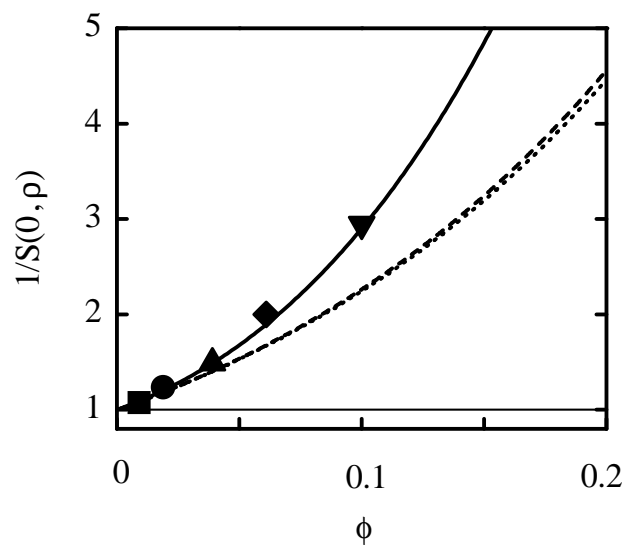

FIG. 5: Inverse structure factor $S^{-1}(q=0, \rho)$ extrapolated to vanishing scattering vectors of stilbenoid dendrimers of the third generation ${ }^{14}$ (with the same symbol code as in Fig. 4 (b)). The dashed line follows from the scaled particle theory according to Eqs. (10), (12), and (13) with $B_{3}^{(S P T)}=16.952$ while the dotted line represents the results of the fundamental measure theory as obtained from Eqs. (10), (12), and (14) with $B_{3}^{(F M T)}=16.432$. The solid line shows the results as obtained from Eqs. (10) and (12) with $B_{3}=34.129$.

In the framework of both scaled particle theory and Rosenfeld's fundamental measure theory $S^{-1}(q=0, \rho)$ is also given by Eqs. (10) - (12) but the third virial coefficient is given by

$$
B_{3}^{(S P T)}=\frac{1}{V_{p}^{2}}\left(V_{p}^{2}+2 \tilde{R}_{p} A_{p} V_{p}+\frac{1}{3} \tilde{R}_{p}^{2} A_{p}^{2}\right)
$$

within scaled particle theory and

$$
B_{3}^{(F M T)} \frac{1}{V_{p}^{2}}\left(V_{p}^{2}+2 \tilde{R}_{p} A_{p} V_{p}+\frac{1}{12 \pi} A_{p}^{3}\right)
$$

within fundamental measure theory. In Fig. 5 the experimentally determined inverse structure factor $S^{-1}(q=0, \rho)$ extrapolated to vanishing scattering vectors of stilbenoid dendrimers of the third generation is compared with the results of scaled particle theory according to Eqs. (10), (12), and (13) and fundamental measure theory according to Eqs. (10), (12), and (14). With increasing volume fraction the theoretical results of both scaled particle theory (dashed line) and fundamental measure theory (dotted line) deviate from the experimental data (symbols). These deviations are mainly due to the fact that the predicted third virial coefficients $B_{3}^{(S P T)}=16.952$ and $B_{3}^{(F M T)}=16.432$ are too small. The results for $S^{-1}(q=0, \rho)$ as obtained from Eqs. (10) and (12) with $B_{3}=34.129$ as input are in agreement

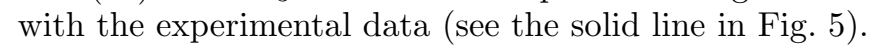
We emphasize that the PRISM theory discussed above leads to a similar agreement with the experimental data as is apparent from Fig. 4 (b).

Our first comparison of experimentally determined $S^{-1}(q=0, \rho)$ of platelike particles with the predictions of 
the well-known scaled particle and fundamental measure theory confirms earlier caveats concerning the applicability of these theories to freely rotating non-spherical particles.

\section{CONCLUSION}

We have presented a systematic application of the PRISM integral equation theory, scaled particle theory, and fundamental measure theory to rigid dendrimers in solution. The main findings and conclusions may be summarized as follows.

PRISM theory is quite successful in reproducing experimental structure factors $S(q, \rho)$ of both polyphenylene dendrimers of the fourth generation [Fig. 4 (a)] and stilbenoid dendrimers of the third generation [Fig. 4(b)], provided the correct form factor $P(q)$ [Fig. 2] is used as input into the generalized Ornstein-Zernike equation of the PRISM [Eq. (8)]. These investigations encourage to pursue a study of charged dendrimers $61,62,63$ within the framework of PRISM by using interaction sites which carry charges.

The inverse structure factor $S^{-1}(q \rightarrow 0, \rho)$ extrapolated to vanishing scattering vectors as predicted by both scaled particle theory and fundamental measure theory is systematically smaller than the experimental data for platelike stilbenoid dendrimers of the third generation and the prediction of the PRISM integral equation theory [Fig. 15 . The substantial differences observed between the experimental data and the results of scaled particle theory and fundamental measure theory are mainly due the fact the both theories do not yield the correct third virial coefficient in the case of platelike particles. Hence there is a clear need to improve both scaled particle theory and fundamental measure theory for freely rotating non-spherical particles.
1 M. Fischer and F. Vögtle, Angew. Chem. Int. Ed. 38, 884 (1999).

2 M. Ballauff, Top. Curr. Chem. 212, 177 (2001).

3 M. Ballauff and C. N. Likos, Angew. Chem. Int. Ed. 43, 2998 (2004).

${ }^{4}$ C. N. Likos and M. Ballauff, Top. Curr. Chem. 245, 239 (2005).

5 H. M. Harreis, C. N. Likos, and M. Ballauff, J. Chem. Phys. 118, 1979 (2003).

${ }^{6}$ K. Müllen, A. J. Berresheim, and M. Müller, Chem. Rev. 99, 1747 (1999).

7 S. Rosenfeldt, N. Dingenouts, D. Pötschke, M. Ballauff, A. J. Berresheim, K. Müllen, and P. Lindner, Angew. Chem. 116, 111 (2004); Angew. Chem. Int. Ed. 43, 109 (2004).

8 S. Rosenfeldt, N. Dingenouts, D. Pötschke, M. Ballauff, A. J. Berresheim, K. Müllen, P. Lindner, and K. Saalwächter, J. Lumin. 111, 225 (2005)

9 M. Wind, K. Saalwächter, U.-M. Wiesler, K. Müllen, and H. W. Spiess, Macromolecules 35, 10071 (2002).

10 P. Carbone, A. Calabretta, M. D. Stefano, F. Negri, and K. Müllen, J. Phys. Chem. A 110, 2214 (2006).

11 H. Meier and M. Lehmann, Angew. Chem. 110, 666 (1998); Angew. Chem. Int. Ed. 37, 643 (1998).

12 H. Meier, M. Lehmann, and U. Kolb, Chem. Eur. J. 6, $2462(2000)$.

13 H. Meier, E. Karpuk, M. Lehmann, D. Schollmeyer, and V. Enkelmann, Z. Naturforsch. B 58, 775 (2003).

14 S. Rosenfeldt, E. Karpuk, M. Lehmann, H. Meier, P. Lindner, L. Harnau, and M. Ballauff, ChemPhysChem 7, 2097 (2006).

15 J. S. Higgins and H. C. Benoit, Polymers and Neutron Scattering, Clarendon, Oxford, (1994).

16 D. Chandler and H. C. Andersen, J. Chem. Phys. 57, 1930 (1972).

17 D. Chandler in Studies in Statistical Mechanics, edited by J. L. Lebowitz and E. W. Montroll, Vol. 8, p. 275, North Holland, Amsterdam (1982).

18 J.-P. Hansen and I. R. McDonald, Theory of Simple Liquids, 2nd edition, Academic Press, London (1986).
19 P. A. Monson and G. P. Morriss, Adv. Chem. Phys. 77, 451 (1990).

20 K. S. Schweizer and J. G. Curro, Phys. Rev. Lett. 58, 246 (1987).

21 A. Yethiraj and C.-Y. Shew, Phys. Rev. Lett. 77, 3937 (1996).

22 A. Yethiraj and C.-Y. Shew, J. Chem. Phys. 106, 5706 (1997).

23 C.-Y. Shew and A. Yethiraj, J. Chem. Phys. 109, 5162 (1998).

24 L. Harnau and P. Reineker, J. Chem. Phys. 112, 437 (2000).

25 L. Harnau, D. Costa, and J.-P. Hansen, Europhys. Lett. 53, 729 (2001).

${ }^{26}$ L. Li, L. Harnau, S. Rosenfeldt, and M. Ballauff, Phys. Rev. E 72, 051504 (2005).

27 C. H. M. Weber, A. Chiche, G. Krausch, S. Rosenfeldt, M. Ballauff, I. Götter-Schnetmann, Q. Tong, S. Mecking, and L. Harnau, Nanoletters, in press (2007).

28 For a review see K. S. Schweizer and J. G. Curro, Adv. Chem. Phys. 98, 1 (1997).

29 L. Harnau, J. Chem. Phys. 115, 1943 (2001).

30 L. Harnau and J.-P. Hansen, J. Chem. Phys. 116, 9051 (2002).

31 S. Bolisetty, C. Airaud, Y. Xu, A. H. E. Müller, L. Harnau, S. Rosenfeldt, P. Lindner, and M. Ballauff, Phys. Rev. E 75, 040803(R) (2007).

32 D. Costa, J.-P. Hansen, and L. Harnau, Mol. Phys. 103, 1917 (2005).

33 H. Reiss, H. L. Frisch, and J. L. Lebowitz, J. Chem. Phys. 31, 369 (1959).

34 H. Reiss, H. L. Frisch, E. Helfand, and J. L. Lebowitz, J. Chem. Phys. 32, 119 (1960).

35 J. L. Lebowitz, E. Helfand, and E. Praestgaard, J. Chem. Phys. 43, 4972 (1965).

36 M. A. Cotter in Molecular Physics of Liquid Crystals, edited G. R. Luckhurst and G. W. Gray, p. 169, Academic, London (1979).

37 B. M. Mulder and D. Frenkel, Mol. Phys. 55, 119 (1985). 
38 S. M. Oversteegen and R. Roth, J. Chem. Phys. 122, 214502 (2005).

39 K. L. Savithramma and N. V. Madhusudana, Mol. Cryst. Liq. Cryst., 74, 243 (1981).

40 T. Boublik, J. Chem. Phys. 63, 4084 (1975).

41 D. Frenkel and R. Eppenga, Phys. Rev. E 49, 1089 (1982).

42 R. Eppenga and D. Frenkel, Mol. Phys. 52, 1303 (1984).

43 J. A. C. Veerman and D. Frenkel, Phys. Rev. A 45, 5632 (1992).

44 M. A. Bates and D. Frenkel, J. Chem. Phys. 110, 6553 (1999).

45 S.-D. Zhang, P. A. Reynolds, and J. S. van Duijneveldt, Mol. Phys. 18, 3041 (2002).

46 S.-D. Zhang, P. A. Reynolds, and J. S. van Duijneveldt, J. Chem. Phys. 117, 9947 (2002).

47 D. van Beek, T. Schilling, and H. N. W. Lekkerkerker, J. Chem. Phys. 121, 5423 (2004).

48 L. Onsager, Phys. Rev. 62, 558, (1942).

49 L. Onsager, Ann. (N.Y.) Acad. Sci. 51, 627 (1949).

50 J. D. Parsons, Phys. Rev. A 19, 1225 (1979).

51 S. D. Lee, J. Chem. Phys. 87, 4972 (1987).

52 S. D. Lee, J. Chem. Phys. 89, 7036 (1989).

${ }^{53}$ H. H. Wensink, Liquid Crystal Phase Behavior of Collodial
Mixtures, PhD thesis, University of Utrecht.

${ }^{54}$ H. N. W. Lekkerkerker, W. C. K. Poon, P. N. Pusey, A. Stoobants, and P. Warren, Europhys. Lett. 20, 559 (1992).

55 Y. Rosenfeld, J. Chem. Phys. 89, 4272 (1988).

56 P. Tarazona and Y. Rosenfeld, Phys. Rev. E 55, R4873 (1997).

57 B. Barboy and W. M. Gelbart, J. Chem. Phys. 71, 3053 (1979).

58 B. Barboy and W. M. Gelbart, J. Stat. Phys. 22, 709 (1980).

59 L. Harnau, D. G. Rowan, and J.-P. Hansen, J. Chem. Phys. 117, 11359 (2002).

60 L. Harnau and S. Dietrich in Soft Matter, edited G. Gompper and M. Schick, Vol. 3 p. 159, Wiley-VCH, Berlin (2007).

61 G. Nisata, R. Ivkov, and E. J. Amis, Macromolecules 33, $4172(2000)$.

62 A. Ohshima, T. Konishi, J. Yamanaka, and N. Ise, Phys. Rev. E, 64, 051808 (2001).

63 A. Ramzi, R. Scherrenberg, J. Joosten, P. Lemstra, and K. Mortensen, Macromolecules 35, 827 (2002). 